\title{
Neutron Lifetime and Axial Coupling Connection
}

\author{
Andrzej Czarnecki \\ Department of Physics, University of Alberta, Edmonton, Alberta, Canada T6G 2E1 \\ William J. Marciano \\ Department of Physics, Brookhaven National Laboratory, Upton, New York 11973, USA \\ Alberto Sirlin \\ Department of Physics, New York University, 726 Broadway, New York, New York 10003, USA
}

(Received 22 February 2018; published 16 May 2018)

\begin{abstract}
Experimental studies of neutron decay, $n \rightarrow p e \bar{\nu}$, exhibit two anomalies. The first is a 8.6(2.1) s, roughly $4 \sigma$ difference between the average beam measured neutron lifetime, $\tau_{n}^{\text {beam }}=888.0(2.0) \mathrm{s}$, and the more precise average trapped ultracold neutron determination, $\tau_{n}^{\text {trap }}=879.4(6) \mathrm{s}$. The second is a $5 \sigma$ difference between the pre2002 average axial coupling, $g_{A}$, as measured in neutron decay asymmetries $g_{A}^{\text {pre2002 }}=1.2637(21)$, and the more recent, post2002, average $g_{A}^{\text {post2002 }}=1.2755(11)$, where, following the UCNA Collaboration division, experiments are classified by the date of their most recent result. In this Letter, we correlate those $\tau_{n}$ and $g_{A}$ values using a (slightly) updated relation $\tau_{n}\left(1+3 g_{A}^{2}\right)=5172.0(1.1) \mathrm{s}$. Consistency with that relation and better precision suggest $\tau_{n}^{\text {favored }}=879.4(6) \mathrm{s}$ and $g_{A}^{\text {favored }}=1.2755(11)$ as preferred values for those parameters. Comparisons of $g_{A}^{\text {favored }}$ with recent lattice QCD and muonic hydrogen capture results are made. A general constraint on exotic neutron decay branching ratios, $<0.27 \%$, is discussed and applied to a recently proposed solution to the neutron lifetime puzzle.
\end{abstract}

DOI: 10.1103/PhysRevLett.120.202002

The neutron lifetime, $\tau_{n}$, and its axial-current coupling, $g_{A}=G_{A} / G_{V}$, are important weak interaction parameters used in nuclear, particle, and astrophysics, as well as cosmology [1-6]. Employed together, they can determine the quark mixing matrix element $V_{\text {ud }}$, at a level that could eventually become competitive with the current superallowed Fermi transition nuclear beta decay method for determining $V_{\text {ud }}$ [7] and constraining "New Physics" via Cabibbo-Kobayashi-Maskawa (CKM) unitarity $\left|V_{\text {ud }}\right|^{2}+$ $\left|V_{\mathrm{us}}\right|^{2}+\left|V_{\mathrm{ub}}\right|^{2}=1$. Neutron decays have the advantage of no nuclear physics uncertainties [8].

On its own, $g_{A}$ provides necessary input for the Goldberger-Treiman relation, the Bjorken sum rule, solar and reactor neutrino fluxes, neutrino-nucleon quasielastic scattering cross sections, muon capture rates, and various other weak interaction phenomena. An area of particular importance is the dependence of primordial nucleosynthesis and cosmic microwave background anisotropies on $\tau_{n}$ and $g_{A}[9,10]$.

Published by the American Physical Society under the terms of the Creative Commons Attribution 4.0 International license. Further distribution of this work must maintain attribution to the author(s) and the published article's title, journal citation, and DOI. Funded by SCOAP ${ }^{3}$.
Despite their central role in weak interaction phenomenology, $\tau_{n}$ and $g_{A}$ values have changed, sometimes dramatically, with time. Indeed, the accepted $\tau_{n}$ has decreased over the Particle Data Group (PDG) lifespan from about $1000 \mathrm{~s} \rightarrow 932 \mathrm{~s} \rightarrow 917 \mathrm{~s} \rightarrow 896 \mathrm{~s} \rightarrow 886 \mathrm{~s}$ while over a similar time span, $g_{A}$ has increased from roughly $1.20 \rightarrow 1.23 \rightarrow 1.25 \rightarrow 1.26 \rightarrow 1.27$. The correlated movement with time of $\tau_{n}$ and $g_{A}$ is nicely illustrated in the introduction figures of Ref. [7]. As we shall argue in this Letter, further change in both quantities appears to be in progress. Although the most precise $\tau_{n}$ and $g_{A}$ experimental measurements have generally been carried out independently of one another, prevailing values at a given time were known to be correlated through the relationship $\tau_{n}\left(1+3 g_{A}^{2}\right)=$ constant, with the constant determined by the standard model (SM) neutron decay rate prediction. Thus, $\tau_{n}$ and $g_{A}$ experimental values can be expected to move together. Here, we review and update (very slightly) the origin, uncertainty, and status of that constant, by updating the inputs, checking the analysis, and assigning an uncertainty to the theory prediction.

Currently, there are two competing values for $\tau_{n}$ and two for $g_{A}$ (see Table I). Although the values in each set are generally averaged by the PDG with errors increased by a scale factor based on the $\chi^{2}$, we keep them separate. The average beam measurements $\tau_{n}^{\text {beam }}=888.0(2.0)$ s differ by about $4 \sigma$ from the newer, more precise, ultracold trapped 
TABLE I. Input data used for the $\tau_{n}^{\text {trap }}, \tau_{n}^{\text {beam }}, g_{A}^{\text {post2002 }}$ and $g_{A}^{\text {pre2002 }}$ averages. Values and methodology were based on PDG2016 but with updates from [12-15]. The error in $\tau_{n}^{\text {trap }}$ average was scaled by a factor of 1.5 in accordance with PDG protocol. Statistical and systematic uncertainties were added in quadrature and kept to two significant figures before averaging. Averages have not been sanctioned by the PDG.

\begin{tabular}{lc}
\hline \hline$\tau_{n}^{\text {trap }}$ & Source \\
\hline $881.5(0.92) \mathrm{s}$ & {$[12]$} \\
$877.7(0.76) \mathrm{s}$ & {$[13]$} \\
$878.3(1.9) \mathrm{s}$ & {$[14]$} \\
$880.2(1.2) \mathrm{s}$ & {$[16]$} \\
$882.5(2.1) \mathrm{s}$ & {$[17]$} \\
$880.7(1.8) \mathrm{s}$ & {$[18]$} \\
$878.5(0.76) \mathrm{s}$ & {$[19]$} \\
$882.6(2.7) \mathrm{s}$ & {$[20]$} \\
$879.4(6) \mathrm{s}$ & Average (includes \\
& scale factor $S=1.5)$ \\
$\tau_{n}^{\text {beam }}$ & Source \\
$887.7(2.2) \mathrm{s}$ & {$[21]$} \\
$889.2(4.9) \mathrm{s}$ & {$[22]$} \\
$888.0(2.0) \mathrm{s}$ & Average \\
$g_{A}^{\text {post2002 }}$ & \\
$1.2772(20)$ & Source \\
$1.2748_{-14}^{+13}$ & {$[15]$} \\
$1.2750(160)$ & {$[23]$} \\
$1.2755(11)$ & {$[24]$} \\
$g_{A}^{\text {pre2002 }}$ & Average \\
$1.2686(47)$ & Source \\
$1.2660(40)$ & {$[25]$} \\
$1.2594(38)$ & {$[26]$} \\
$1.2620(50)$ & {$[27]$} \\
$1.2637(21)$ & {$[28]$} \\
\hline \hline
\end{tabular}

neutron average $\tau_{n}^{\text {trap }}=879.4(6) \mathrm{s}$. That difference is sometimes referred to as the neutron lifetime puzzle, enigma, or problem. Similarly, an earlier set of $g_{A}$ measurements labeled pre2002 averages to $g_{A}^{\text {pre2002 }}=$ 1.2637(21), while determinations completed after 2002, labeled post2002, average to $g_{A}^{\text {post2002 }}=1.2755(11)$, a $5 \sigma$ difference, even more pronounced than the neutron lifetime problem. A notable difference $[1,11]$ between pre and post 2002 experiments, is that the earlier efforts required larger corrections to the measured asymmetries. As a result, those corrections and their systematic uncertainties may have been more difficult to properly estimate. The two $g_{A}$ values are generally PDG averaged, and the uncertainty is increased by a scale factor of approximately 2 , primarily due to pre $2002 \chi^{2}$ contributions. Here, we keep the method dependent $\tau_{n}$ as well as the pre and post $2002 g_{A}$ values separate, and we argue in favor of the more recent values in both cases because of their better precision and, more important, their remarkable consistency with our evaluation of the constant in the $\tau_{n}-g_{A}$ relation previewed above. On that basis, we will argue that, within the SM, $\tau_{n}^{\text {favored }}=$ $879.4(6) \mathrm{s}$ and $g_{A}^{\text {favored }}=1.2755(11)$ currently represent our recommended "favored values." They may be the final word, within errors.

Relating $\tau_{n}$ and $g_{A}$ begins with a very precise SM prediction for the total (radiative inclusive) neutron decay rate. That inverse lifetime formula includes Fermi function final state electron-proton Coulomb interactions, electroweak radiative corrections (normalized relative to the muon lifetime [29]), and a number of smaller effects including proton recoil, finite nuclear size etc. Overall, those corrections are rather large, $>+7 \%$. A very detailed analysis of those corrections was given in the classic study by Wilkinson [30]. Later, that relationship was checked, updated, and refined in [31] where higher order $\mathcal{O}\left(\alpha^{2}\right)$ contributions were properly included. The radiative corrections uncertainty was reduced in [32].

In the SM, the inverse lifetime equation relating $\tau_{n}$ and $g_{A}$ is given by [31]

$$
\frac{1}{\tau_{n}}=\frac{G_{\mu}^{2}\left|V_{\mathrm{ud}}\right|^{2}}{2 \pi^{3}} m_{e}^{5}\left(1+3 g_{A}^{2}\right)(1+\mathrm{RC}) f,
$$

where $G_{\mu}$ is the Fermi constant determined from the muon lifetime [33-42], $G_{\mu}=1.1663787(6) \times 10^{-5} \mathrm{GeV}^{-2}, V_{\text {ud }}$ is the CKM mixing element generally obtained from superallowed nuclear beta decays [7,43], RC represents electroweak radiative corrections [44-50], which were most recently evaluated [32] to be $+0.03886(38)$, and $f$ is a phase space factor [30]. The electroweak radiative corrections in Eq. (1) have been factorized to be the same for vector and axial-vector contributions [31]. That prescription defines $g_{A}$ as determined by the neutron lifetime. Expressing the polarized neutron spin-electron correlation coefficient, $A_{0}\left(g_{A}\right)=2 g_{A}\left(1-g_{A}\right) /\left(1+3 g_{A}^{2}\right)$, in terms of that $g_{A}$ will, therefore, induce small $\mathcal{O}(0.1 \%)$ radiative corrections [51] along with the $\mathcal{O}(1 \%)$ residual Coulomb, recoil, and weak magnetism corrections to the measured asymmetry that must be corrected for before extracting $g_{A}[5,30]$.

Employing masses [7] (with highly correlated uncertainties due to atomic mass units to $\mathrm{MeV}$ translation) $m_{n}=$ 939.5654133(58) MeV, $\quad m_{p}=938.2720813(58) \mathrm{MeV}$, and $m_{e}=0.5109989461(31) \mathrm{MeV}$ leads to $f=1.6887(1)$ [30,31], where we have redone the numerical evaluation of Wilkinson's perturbative analysis and employed a conservative error consistent with his assessment [52]. Using the above input parameters, but keeping $V_{\mathrm{ud}}, \tau_{n}$ and $g_{A}$ arbitrary, produces the SM master formula

$$
\left|V_{\mathrm{ud}}\right|^{2} \tau_{n}\left(1+3 g_{A}^{2}\right)=4908.6(1.9) \mathrm{s},
$$

where the uncertainty comes primarily from the RC. That formula can be used to determine $V_{\text {ud }}$ from independent experimental measurements of $\tau_{n}$ and $g_{A}$. Future experiments 
optimistically hope to eventually reach $\pm 0.01 \%$ sensitivity for those input parameters. At that level, the RC theory uncertainty will be dominant.

Our intention is to correlate $\tau_{n}$ and $g_{A}$, rather than determine $V_{\text {ud }}$. To that end, we employ the superallowed $0^{+} \rightarrow 0^{+}$nuclear transitions current best value $V_{\text {ud }}=$ $0.97420(10)(18)_{\mathrm{RC}}$, a value consistent with CKM unitarity [7], where the first error (10) results from experiment, nuclear structure, and nucleus dependent radiative corrections, while the second error $(18)_{\mathrm{RC}}$ represents universal radiative corrections common to both neutron and nuclear beta decays. Importantly, the RC error in $\left|V_{\mathrm{ud}}\right|^{2}$ and in Eq. (1) are anticorrelated and effectively cancel. For that reason, one finds the following very precise relation between $\tau_{n}$ and $g_{A}$

$$
\tau_{n}\left(1+3 g_{A}^{2}\right)=5172.0(1.1) \mathrm{s},
$$

where the uncertainty stems primarily from nuclear and experimental uncertainties in $V_{\text {ud }}$. That connection allows one to translate between $\tau_{n}$ and $g_{A}$ with high precision and thereby test their mutual consistency.

In that way, lifetime and axial-charge measurements can be directly compared or, for some purposes, even averaged. Toward that end, it is useful to divide the lifetime averages into trap, which includes bottle and magnetic confinement trap experiments, and beam measurements, the two areas of disagreement. Similarly, following the classification introduced by the UCNA Collaboration [15], asymmetry values of $g_{A}$ naturally separate into pre2002 and post2002, where 2002 represents the year when larger values of $g_{A}$, seen earlier, were confirmed with improved errors [11,53]. Experiments are arranged by the year of their last result (see Table I). The post2002 measurements of $g_{A}$ tended to have larger central values and better controlled systematics. That approach leads to the following direct and indirect averages, connected by arrows representing the relationship in Eq. (3),

$$
\begin{gathered}
\tau_{n}^{\text {trap }}=879.4(6) \mathrm{s} \rightarrow g_{A}=1.2756(5), \\
\tau_{n}^{\text {beam }}=888.0(2.0) \mathrm{s} \rightarrow g_{A}=1.2681(17), \\
g_{A}^{\text {post2002 }}=1.2755(11) \rightarrow \tau_{n}=879.5(1.3) \mathrm{s}, \\
g_{A}^{\text {pre2002 }}=1.2637(21) \rightarrow \tau_{n}=893.1(2.4) \mathrm{s} .
\end{gathered}
$$

One notices that $\tau_{n}^{\text {trap }}$ and $g_{A}^{\text {post2002 }}$ provide the most precise direct and indirect lifetimes, respectively, and they are remarkably consistent. Those features are illustrated in Fig. 6 of Ref. [15]. That agreement is exactly the type of consistency one expects of the true parameters. On the other hand, the beam and pre2002 $g_{A}$ determined lifetimes disagree with those more precise values and are not particularly consistent with one another. Because of their better precision and relationship consistency, we refer to the trap lifetime and post2002 $g_{A}$ as our favored values,

$$
\begin{gathered}
\tau_{n}^{\text {favored }}=879.4(6) \mathrm{s}, \\
g_{A}^{\text {favored }}=1.2755(11) .
\end{gathered}
$$

These favored experimental averages, in conjunction with the indirect determination of $g_{A}$ in Eq. (4), provide standards for comparison with future lifetime and asymmetry measurements which will aim at the long term goal of $0.01 \%$ precision in $\tau_{n}$ and $g_{A}$. Our current favored values in Eqs. (8) and (9) should be compared with our updates of the 2016 PDG averages based on recent results [12-15] in Table I,

$$
\begin{gathered}
\tau_{n}^{\text {update } 16}=879.7(8) \mathrm{s} \quad(\text { with scale factor } S=2), \\
g_{A}^{\text {update } 16}=1.2731(23) \quad(\text { with } S=2.3) .
\end{gathered}
$$

Those updates are consistent with our preferred values in Eqs. (8), (9), but they have larger errors due to scale factors that represent inconsistencies in the experiments averaged. They are useful as a conservative perspective on the current $\tau_{n}$ and $g_{A}$ situation.

Regarding our neglect of $\tau_{n}^{\text {beam }}$ and $g_{A}^{\text {pre2002 }}$ in deriving our favored values, we make the following observations. $\tau_{n}^{\text {beam }}$ differs from $\tau_{n}^{\text {trap }}$ by about $4 \sigma$ and $g_{A}^{\text {pre2002 }}$ differs from $g_{A}^{\text {post2002 }}$ by $5 \sigma$. So, a case can be made that one should not continue to include in averaging outlying values based on older techniques when a significant disagreement arises. Indeed, the history of $\tau_{n}$ and $g_{A}$ experimental shifts indicate that they come in pairs as new technological methods emerge. In this case, the 2002 confirmation [11,53] of a relatively large $g_{A}$ with small errors may be viewed as the harbinger of a shorter lifetime, which several years later began to be directly observed in trapped lifetime experiments.

One might ask whether theory or some other weak interaction phenomenon can be used to determine $g_{A}$ (and $\tau_{n}$ indirectly)? On the theory side, there is the promise of lattice QCD [54,55]. The lattice approach is, in principle, an ideally suited first principles method for computing a relatively pure, strong interaction effect such as $g_{A}$. However, early lattice attempts to compute $g_{A}$ generally obtained smaller than expected values with large systematic uncertainties. Recently, the situation has been improving. Indeed, a recent study [56] found the preliminary result, $g_{A}^{\text {lattice }}=1.285(17)$, in good agreement with our "favored" value at about the $\pm 1 \%$ level. How much further the lattice precision can improve remains to be seen. Fortunately, the current uncertainty is statistics dominated; so, long dedicated lattice running can potentially reduce the error. Perhaps our suggestion of a favored value with small uncertainty may help to motivate a heroic effort.

An alternative independent experimental $g_{A}$ determination using muon capture in Muonic Hydrogen was recently examined [57]. Using theory and experimental input for 
other parameters, the measured capture rate gave $g_{A}=$ $1.276(11)$ i.e. somewhat better than $1 \%$ agreement with our favored value. It was suggested in that study that a future factor of 3 improvement in the measured capture rate combined with a better lattice determination of the axial charge radius could provide a $g_{A}$ determination at the level of $\pm 0.2-0.3 \%$. That would be a nice check on our favored values, but it would appear difficult to improve that approach much further.

To illustrate an application of the favored values, we end our discussion by deriving a general constraint on possible exotic (beyond the standard model) neutron decays and applying it to an interesting scenario recently put forward by Fornal and Grinstein (FG) [58] in an effort to solve the neutron lifetime puzzle. Those authors suggest that the $\mathrm{BR}=$ branching ratio for radiative inclusive $n \rightarrow p e \bar{\nu}(\gamma)$ could be 0.99 rather than 1 due to a speculated $1 \%$ exotic neutron branching ratio into dark particle decay modes (e.g., $n \rightarrow$ dark $n+$ scalar) without protons and electrons. In that case, beam experiments that only detect decays with final state protons or electrons would actually measure a partial lifetime $\tau_{n}^{\text {full }} / \mathrm{BR}$ with $\mathrm{BR}<1$, while trapped neutron experiments that count the number of neutrons as a function of time measure the full inclusive lifetime $\tau_{n}^{\text {full }}$. Although throughout this Letter, we tacitly conclude that the beam lifetime is an outlier whose value will shift in future, more precise, follow-up experiments, and eventually agree with our favored trapped $\tau_{n}^{\text {trap }}=879.4(6) \mathrm{s}$, addressing the Fornal-Grinstein solution is an instructive exercise that we will use to conclude this Letter.

We begin by generalizing our analysis to the case where the BR for $n \rightarrow p e \bar{\nu}(\gamma)$ can be $<1$ due to exotic decays, such as $n \rightarrow$ dark particles. In that case, Eqs. (1), (2), and (3) are modified by replacing $\tau_{n}$ with $\tau_{n}^{\text {full }} / \mathrm{BR}$, where $\tau_{n}^{\text {full }}=1 /$ (total nentron decay rate). That replacement leads, via Eq. (3), to (assuming $V_{\text {ud }}$ extracted from superallowed beta decays and CKM unitarity agreement are negligibly affected by the exotic new physics)

$$
\mathrm{BR}=\tau_{n}^{\text {full }}\left(1+3 g_{A}^{2}\right) / 5172.0(1.1) \mathrm{s} .
$$

Accepting $\tau_{n}^{\text {trap }}=879.4(6) \mathrm{s}$ as the full lifetime in Eq. (12) and expanding BR in $g_{A}$ about $g_{A}=1.2755$, the directly measured axial coupling post2002 central value, leads to

$$
\mathrm{BR}=0.9999(7)+1.30\left(g_{A}-1.2755\right)+\ldots
$$

That formula demonstrates the closeness of BR to 1 for $g_{A}^{\text {favored }}=1.2755(11)$. It suggests a degree of tension between the recent determinations of $g_{A}^{\text {post2002 }}$ and the Fornal-Grinstein solution to the neutron lifetime puzzle. In fact, phrased as a one sided 95\% C.L. bound, it requires

$1-\mathrm{BR}=$ Total exotic neutron decay branching ratio

$$
<0.27 \% \text { for } g_{A}=1.2755(11) \text {. }
$$

That bound implies that satisfying more than $2.4 \mathrm{~s}$ of the $8.6 \mathrm{~s}$ lifetime puzzle difference has less than a 5\% chance of being realized. One can overcome such a likelihood restriction by assuming a smaller $g_{A}$ in Eq. (13). For example, $g_{A}=1.268$ leads to $\mathrm{BR}=0.99$, which corresponds to about a $9 \mathrm{~s}$ lifetime difference. Any axial coupling roughly in the range $1.268<g_{A}<1.272$ could account for a good part of the puzzle. Unfortunately, there would be a price to pay for a smaller $g_{A}$ in that range. Those values are in disagreement with the most recent $g_{A}^{\text {post2002 }}=$ $1.2755(11)$ by 3 or more $\sigma$. Thus, the lifetime puzzle would be replaced by a $g_{A}$ inconsistency.

The Fornal-Grinstein scenario will be tested by new measurements of $\tau_{n}$, both beam and trap, to see if the current puzzle survives and needs a solution. If so, the next step will be more precise determinations of $g_{A}$, via neutron decay asymmetries or perhaps lattice gauge theories. Will $g_{A}$ revert back to a smaller value? Updates of $g_{A}$ in the past have almost always led to larger values, but the past is not always a good predictor for the future.

A scenario similar to that of Fornal and Grinstein was envisioned by K. Green and D. Thompson [59] for the rare decay $n \rightarrow$ hydrogen $+\bar{\nu}$. They used the different effects of that decay on beam and trap lifetimes to obtain a bound of $<3 \%$ for that branching ratio (to be compared with the $4 \times 10^{-6}$ prediction [60-63]). Our general analysis employing $\tau_{n}^{\text {trap }}$ and $g_{A}^{\text {post2002 }}$ in Eq. (12) can be used to reduce that bound by an order of magnitude to $<0.27 \%$.

Our $0.27 \%$ bound in Eq. (14) also applies to neutron oscillations into mirror or dark neutrons [64-66], exotic phenomena proposed to explain the neutron lifetime puzzle.

Future expected order of magnitude improvements in $\tau_{n}^{\text {trap }}$ and asymmetry measurements, should improve the sensitivity of our bound in Eq. (14) to roughly $3 \times 10^{-4}$ for the exotic phenomena described above.

The work of A. C. was supported by the Natural Sciences and Engineering Research Council of Canada. The work of W. J. M. was supported by the U.S. Department of Energy under Grant No. DE-SC0012704. The work of A. S. was supported in part by the National Science Foundation under Grant No. PHY-1620039.

[1] H. Abele, Prog. Part. Nucl. Phys. 60, 1 (2008).

[2] D. Dubbers and M. G. Schmidt, Rev. Mod. Phys. 83, 1111 (2011).

[3] F. E. Wietfeldt and G. L. Greene, Rev. Mod. Phys. 83, 1173 (2011).

[4] N. Severijns and O. Naviliat-Cuncic, Annu. Rev. Nucl. Part. Sci. 61, 23 (2011).

[5] A. N. Ivanov, M. Pitschmann, and N. I. Troitskaya, Phys. Rev. D 88, 073002 (2013).

[6] G. L. Greene and P. Geltenbort, Sci. Am. 314, 36 (2016).

[7] C. Patrignani et al. (Particle Data Group), Chin. Phys. C 40, 100001 (2016). 
[8] H. Abele, E. Barberio, D. Dubbers, F. Gluck, J. C. Hardy, W. J. Marciano, A. Serebrov, and N. Severijns, Eur. Phys. J. C 33, 1 (2004).

[9] G. J. Mathews, T. Kajino, and T. Shima, Phys. Rev. D 71, 021302 (2005).

[10] C. Pitrou, A. Coc, J.-P. Uzan, and E. Vangioni, arXiv: 1801.08023.

[11] H. Abele, M. A. Hoffman, S. Baessler, D. Dubbers, F. Gluck, U. Muller, V. Nesvizhevsky, J. Reich, and O. Zimmer, Phys. Rev. Lett. 88, 211801 (2002).

[12] A. P. Serebrov et al., arXiv:1712.05663.

[13] R. W. Pattie, Jr. et al., arXiv:1707.01817.

[14] V. F. Ezhov et al., arXiv:1412.7434.

[15] M. A. P. Brown et al. (UCNA), Phys. Rev. C 97, 035505 (2018).

[16] S. Arzumanov, L. Bondarenko, S. Chernyavsky, P. Geltenbort, V. Morozov, V. V. Nesvizhevsky, Yu. Panin, and A. Strepetov, Phys. Lett. B 745, 79 (2015).

[17] A. Steyerl, J. M. Pendlebury, C. Kaufman, S. S. Malik, and A. M. Desai, Phys. Rev. C 85, 065503 (2012).

[18] A. Pichlmaier, V. Varlamov, K. Schreckenbach, and P. Geltenbort, Phys. Lett. B 693, 221 (2010).

[19] A. Serebrov et al., Phys. Lett. B 605, 72 (2005).

[20] W. Mampe, L. N. Bondarenko, V. I. Morozov, Yu. N. Panin, and A. I. Fomin, Pis'ma Zh. Eksp. Teor. Fiz. 57, 77 (1993) [JETP Lett. 57, 82 (1993)].

[21] A. T. Yue, M. S. Dewey, D. M. Gilliam, G. L. Greene, A. B. Laptev, J. S. Nico, W. M. Snow, and F. E. Wietfeldt, Phys. Rev. Lett. 111, 222501 (2013).

[22] J. Byrne and P. G. Dawber, Europhys. Lett. 33, 187 (1996).

[23] D. Mund, B. Markisch, M. Deissenroth, J. Krempel, M. Schumann, H. Abele, A. Petoukhov, and T. Soldner, Phys. Rev. Lett. 110, 172502 (2013).

[24] M. Schumann, T. Soldner, M. Deissenroth, F. Gluck, J. Krempel, M. Kreuz, B. Markisch, D. Mund, A. Petoukhov, and H. Abele, Phys. Rev. Lett. 99, 191803 (2007).

[25] Yu. A. Mostovoi et al., Yad. Fiz. 64, 2040 (2001) [Phys. At. Nucl. 64, 1955 (2001)].

[26] P. Liaud, K. Schreckenbach, R. Kossakowski, H. Nastoll, A. Bussiere, J. P. Guillaud, and L. Beck, Nucl. Phys. A612, 53 (1997).

[27] B. Erozolimsky, I. Kuznetsov, I. Stepanenko, and Yu. A. Mostovoi, Phys. Lett. B 412, 240 (1997).

[28] P. Bopp, D. Dubbers, L. Hornig, E. Klemt, J. Last, H. Schutze, S. J. Freedman, and O. Scharpf, Phys. Rev. Lett. 56, 919 (1986); 57, 1192(E) (1986).

[29] W. J. Marciano, J. Phys. G 29, 23 (2003).

[30] D. H. Wilkinson, Nucl. Phys. A377, 474 (1982).

[31] A. Czarnecki, W. J. Marciano, and A. Sirlin, Phys. Rev. D 70, 093006 (2004).

[32] W. J. Marciano and A. Sirlin, Phys. Rev. Lett. 96, 032002 (2006).

[33] T. Kinoshita and A. Sirlin, Phys. Rev. 113, 1652 (1959).
[34] S. M. Berman, Phys. Rev. 112, 267 (1958).

[35] S. Berman and A. Sirlin, Ann. Phys. (N.Y.) 20, 20 (1962).

[36] E. S. Abers, D. A. Dicus, R. E. Norton, and H. R. Quinn, Phys. Rev. 167, 1461 (1968).

[37] T. van Ritbergen and R. G. Stuart, Phys. Rev. Lett. 82, 488 (1999).

[38] M. Steinhauser and T. Seidensticker, Phys. Lett. B 467, 271 (1999).

[39] A. Ferroglia, G. Ossola, and A. Sirlin, Nucl. Phys. B560, 23 (1999).

[40] W. J. Marciano, Phys. Rev. D 60, 093006 (1999).

[41] I. Blokland, A. Czarnecki, M. Ślusarczyk, and F. Tkachov, Phys. Rev. Lett. 93, 062001 (2004).

[42] A. Pak and A. Czarnecki, Phys. Rev. Lett. 100, 241807 (2008).

[43] J. Hardy and I. S. Towner, Proc. Sci. CKM2016 (2016) 028.

[44] A. Sirlin, Phys. Rev. 164, 1767 (1967).

[45] A. Sirlin, in Particles, Currents, Symmetries, Acta Physica Austriaca, Suppl. V, edited by P. Urban (Springer Verlag, New York, 1968), p. 353.

[46] A. Sirlin, Nucl. Phys. B71, 29 (1974).

[47] A. Sirlin, Rev. Mod. Phys. 50, 573 (1978).

[48] R. Blin-Stoyle and J. M. Freeman, Nucl. Phys. A150, 369 (1970).

[49] A. Sirlin, AIP Conf. Proc. 698, 385 (2004).

[50] A. Sirlin and A. Ferroglia, Rev. Mod. Phys. 85, 263 (2013).

[51] R. T. Shann, Nuovo Cimento A 5, 591 (1971).

[52] D. H. Wilkinson, Nucl. Instrum. Methods Phys. Res., Sect. A 404, 305 (1998).

[53] H. Abele et al., Phys. Lett. B 407, 212 (1997).

[54] S. Capitani, M. Della Morte, D. Djukanovic, G. M. von Hippel, J. Hua, B. Jäger, P. M. Junnarkar, H. B. Meyer, T. D. Rae, and H. Wittig, arXiv:1705.06186.

[55] E. Shintani and H. Wittig, JPS Conf. Proc. 10, 062001 (2016).

[56] C. C. Chang et al., arXiv:1710.06523.

[57] R. J. Hill, P. Kammel, W. J. Marciano, and A. Sirlin, arXiv: 1708.08462.

[58] B. Fornal and B. Grinstein, Phys. Rev. Lett. 120, 191801 (2018).

[59] K. Green and D. Thompson, J. Phys. 16, L75 (1990).

[60] J. N. Bahcall, Phys. Rev. 124, 495 (1961).

[61] P. K. Kabir, Phys. Lett. 24B, 601 (1967).

[62] J. McAndrew, S. Paul, R. Engels, P. Fierlinger, E. Gutsmiedl, J. Schön, and W. Schott, Phys. Procedia 51, 37 (2014).

[63] R. Daudel, M. Jean, and M. Lecoin, J. Phys. Radium 8, 238 (1947).

[64] Z. Berezhiani and L. Bento, Phys. Rev. Lett. 96, 081801 (2006).

[65] A. P. Serebrov et al., Phys. Lett. B 663, 181 (2008).

[66] A. P. Serebrov, R. M. Samoilov, I. A. Mitropolsky, and A. M. Gagarsky, arXiv:1802.06277. 